\title{
Salinity and Boron Tolerance in Cotton (Gossypium hirsutum) Varieties: A Short-term Hydroponic Study
}

\author{
Muhammad Aftab ${ }^{1 *}$, Muhammad Anwar-ul-Haq ${ }^{1^{*}}$, Javaid Akhtar ${ }^{1}$ and Ejaz Ahmad Waraich ${ }^{2}$ \\ ${ }^{1}$ Institute of Soil and Environmental Sciences, University of Agriculture Faisalabad, Pakistan \\ ${ }^{2}$ Department of Crop Physiology, University of Agriculture Faisalabad, Pakistan \\ *For correspondence: m.aftabjee@gmail.com; haqgondal@gmail.com
}

\begin{abstract}
High salinity in association with boron toxicity is becoming very adverse for crop production in arid to semi arid areas throughout the world. In order to investigate the effect of salinity in combination with boron toxicity on six cotton varieties, a hydroponic study was conducted in the rain protected wire house using $1 / 2$ strength Hoagland's nutrient solution as a growth medium. One week after transplantation, different levels of salinity i.e. 70 and $140 \mathrm{mM}$ were developed with $\mathrm{NaCl}$. The concentration of boron $(5$ and $10 \mathrm{mM})$ was created using boric acid $\left(\mathrm{H}_{3} \mathrm{BO}_{3}\right)$. The plants were harvested after four weeks of salinity and boron toxicity development. The result indicated that the chlorophyll contents in variety FH-114 were less affected against combined stress condition because it accmulated less $\mathrm{Na}^{+}$and boron but acquired more $\mathrm{K}^{+}$which result into batter growth as compared to other varieties whereas NIAB-846 was found very sensitive against combined stress. The trend regarding the varietal behaviour with salinity and boron stress was FH-114 > NIAB-777 > IUB-222 > SG1-AA > FH-113 > NIAB-846 on the basis of this study; the variety FH-114 can be grown in slinity and boron stress conditions because it has potential to regulate growth by regulating $\mathrm{Na}^{+} / \mathrm{K}^{+}$uptake. (C) 2015 Friends Science Publishers
\end{abstract}

Keywords: Abiotic stress; Boron; Cotton; Salinity; $\mathrm{Na}^{+} ; \mathrm{K}^{+}$

\section{Introduction}

Salinity is one of the major abiotic stresses for agriculture all over the world. More than $800 \mathrm{~m}$ ha of land arround the globe is salt affected either by salinity (397 $\mathrm{m} \mathrm{ha})$ or the associated condition of sodicity (434 $\mathrm{m}$ ha). Of the current 230 million ha of irrigated land, 20\% (45 m ha) are saltaffected (FAO, 2008). Decline in agriculture production due to salinity is one of the key problems in many areas around the world including Pakistan (Ashraf and Foolad, 2007). Under salinity stress, plant growth is reduced due to restricted uptake of the essential elements such as $\mathrm{K}^{+}$ (Abbas et al., 2013). Boron (B) is an indispensable micronutrient which is required for plants normal growth (Miwa and Fujiwara, 2010). Different plant species have different $\mathrm{B}$ requirements but its defficiency and toxicity range is very narrow than other nutients. Excessive B problem has been reported in the soils of South Australia, India, Iraq, Pakistan, Peru and USA (Hasnain et al., 2011). In semi arid and arid area where brakish ground water is use for irrigation purpose the B toxicity may occur. Boron chemistry is very simple in soil when it compared with other elements present in soil. The oxidation reduction or volatilization reactions occur in soil does not effect it. Boric acid $\left(\mathrm{H}_{3} \mathrm{BO}_{3}\right)$ is a monobasic, very weak acid that acts as a lewis acid by accepting a hydroxyal ion to form borate anion (Ashraf and Ahmad, 2000). For sustainable crop production in semi arid and arid areas arround the globe B toxicity is a serious concern because good quality irrigation water is inadequate for crop production and formers use brakish water for irrigation purpose in these areas (Saleem et al., 2011). Boron is transported to aerial parts of the plant by transpiration stream through xylem and it accmulate at the tipes and margins of older leaves (Sestren and Kroplin, 2009). In older leaves accmulation of B occur which could imbalance the constituents of cell wall which leads to tissue necrosis and eventually death occur (Sestren and Kroplin, 2009). In case of boron toxicity the concentration of $B$ increases in cytosol, which causes the metabolic dysfunctions by the formation of different complexes with $\mathrm{NAD}^{+}$which affects the structure of RNA (Loomis and Durst, 1992). However in mature tissues toxicity may be due photo-oxidative stress, retarducation of many celluler prossess (Reid et al., 2004). Many celluler processes in vascular plants are negatively affected by B toxicity such as chlorophyll contents in leaf, photosynthesis, cell division of root and levels of subrin and lignin (Reid, 2007). As a result root shoot growth of plants which are exposed to higher level of B is reduced (Nable et al., 1990).

Worldwide cotton is an important cash crop. In 
agriculture based economy of Pakistan it plays a vital role. After China, USA, and India fourth largest cotton producing country is Pakistan (Ahmad et al., 2009). $3.1 \mathrm{~m}$ ha of the Pakistan is under cultivation with the 12.4 million bales of production (Anonymous, 2007). In Pakistan cotton is also an important source of vegetable oil, in addition it produce fiber. Significant foreign exchange it generates. Although cotton is classified as a salinity tolerant crop with threshold EC of $7.7 \mathrm{dS} \mathrm{m}^{-1}$ (Maas and Hoffmann, 1977). However, cotton at seedling emergence stage is adversly affected by salinity (Ashraf, 2002). Under saline conditions, the uptake of $\mathrm{B}$ is enhanced and these conditions are more injurious for crop production (Grieve et al., 2010). To the best of our knowledge, no work has been done regarding the tolerance of cotton against the combination of salinity and B toxicity. Therefore, the present study was conducted to explore the tolerance poential of cotton varieties against combined stress of salinity and B toxicity.

\section{Materials and Methods}

This hydroponic experiment was conducted in the rain protected wire house of the Institute of Soil and Environmental Sciences, University of Agriculture Faisalabad (ISES, UAF), Pakistan. Seeds of six cotton (Gossypium hirsutum L.) varieties (NIAB-846, IUB-222, FH-114, FH-113, NIAB-846, SGI-AA) were collected from different research institutes of the Punjab such as Nuclear Institute of Agriculture and Biology (NIAB) Faisalabad, Islamic University Bahawalpur, Ayub Agriculture Research Institute (AARI) Faisalabad and Ali Akbar Group of Companies. The seeds were sown in trays having two inch layer of sand washed with distilled water. Seeds were kept moistened with water before germination and with $1 / 2$ strength Hoagland's nutrient solution (Hoagland and Arnon, 1950) after seedling emergence. At two leaf stage, the healthy and uniform seedlings were transplanted in foam plugged holes in polystyrene sheets floating over $25 \mathrm{~L}$ capacity plastic tubs containing $1 / 2$ strength of Hoagland's nutrient solution. One week after transplantation, tratments were applied to the plants. Different levels of salinity i.e. 70 and $140 \mathrm{mM}$ were developed with calculated amount of $\mathrm{NaCl}$. The toxicity of $\mathrm{B}(5$ and $10 \mathrm{mM})$ was created using $\mathrm{H}_{3} \mathrm{BO}_{3}$. These levels of $\mathrm{B}$ were maintained on the basis of earlier work by (Ahmad et al., 2005; 2008). Both $\mathrm{NaCl}$ and bori acid were applied in two increments (one per day) whereas no salt was added in control. The solution $\mathrm{pH}$ was maintained at $\sim 6$ with dilute $\mathrm{NaOH}$ and $\mathrm{HCl}$ and the solution was changed weekly during the period of study. Before harvesting, the chlorophyll contents of second top leaf were measured by Minolta-502 chlorophyll meter. After four weeks of transplantation, the plants were harvested. The seprate shoots and roots samples were oven dried at $75^{\circ} \mathrm{C}$ for $48 \mathrm{~h}$. The dry weight of root and shoot were recorded. The dried shoot samples were ground in grinding machine and ashing of grinded samples was done in muffle furnace (E.Y Ela, Electric Furnace, T.M.F. 2100) at $550^{\circ} \mathrm{C}$ for $6 \mathrm{~h}$ (Reuter and Robinson, 1986; Mills and Jones, 1996). The ash was dissolved in $2 \mathrm{mM} \mathrm{HNO}_{3}$ and filtered through Whatman No. 40 filter paper. The volume was made up to $50 \mathrm{~mL}$ with distilled water and used for ionic analysis. The ionic concentration of $\mathrm{Na}^{+}$and $\mathrm{K}^{+}$in samples were determined by using Sherwood-410 Flame Photometer with the help of self prepared standard solutions using reagent grade salts of $\mathrm{NaCl}$ and $\mathrm{KCl}$, respectively. Boron was determined using azomethine- $\mathrm{H}$ as a color developing reagent and read absorbance at $420 \mathrm{~nm}$ on a spectrophotometer (Malekani and Cresser, 1998).

\section{Statistical Analysis}

Data of the experiment were subjected to analysis of variance in a completely randomized design with factorial arrangements (Steel et al., 1997). The significance of differences among treatments and varieties were determined using DMR test.

\section{Results}

\section{Shoot and Root Growth}

Shoot dry weight of all varieties decresed significantly by salinity, boron and their interaction. The effect of treatment, variety and their interaction was significant at $p \leq 0.05$. The interactive effect of both the treatments was more detrimental than the either individual treatment (Table 1). The comparison of varieties indicated that $\mathrm{FH}-114$ produced more shoot dry weight in all the stress treatments compared to all other varieties. The minimum shoot dry weight was produed by NIAB-846. When the higher levels of both the salinity and $\mathrm{B}$ were combined i.e. $140 \mathrm{mM} \mathrm{NaCl}+10 \mathrm{mM}$ $\mathrm{B}$, the percentage reduction in shoot dry weight was 57 and $92 \%$ in both these varities respectively (Table 1). The remaining four varieties (NIAB-777, IUB-222, FH-113 and SG1 AA) produced shoot dry weight in between these two varieties (FH-114 and NIAB-846). Root dry weight was also decreased in response to both stresses with combined effect proved to be more harmful (Table 2). The effect of treatment, variety and their interaction was significant at $\mathrm{p} \leq$ 0.05 . The roots of FH-114 showed more tolerance to salinity and boron stress as compared to other varieties whereas this tolerance was minimum in the case of NIAB-846. The percentage reduction in root dry weight was 63 and $89 \%$ in both these varities respectively, when the higher levels of both the stresses were combined i.e. at $140 \mathrm{mM} \mathrm{NaCl}+10$ $\mathrm{mM}$ B.

\section{Chlorophyll Contents}

Data regarding chlorophyll contents showed that there was significant difference among different cotton varieties at $\mathrm{p} \leq$ 0.05. As salinity and boron stress levels increased 
Salinity and Boron Interaction in Cotton / Int. J. Agric. Biol., Vol. 17, No. 4, 2015

Table 1: Shoot dry weight of different cotton varieties $\left(\mathrm{g}_{\mathrm{glant}}{ }^{-1}\right)$ at various salinity and boron levels

\begin{tabular}{llllllllll}
\hline Varieties & Control & $70 \mathrm{mM} \mathrm{NaCl}$ & $140 \mathrm{mM} \mathrm{NaCl}$ & $5 \mathrm{mM} \mathrm{B}$ & $10 \mathrm{mM} \mathrm{B}$ & $\begin{array}{l}70 \mathrm{mM} \mathrm{NaCl}+5 \\
\mathrm{mM} \mathrm{B}\end{array}$ & $\begin{array}{l}70 \mathrm{mM} \\
\mathrm{NaCl}+10 \mathrm{mM} \mathrm{B}\end{array}$ & $\begin{array}{l}140 \mathrm{mM} \\
\mathrm{NaCl}+5 \mathrm{mM} \mathrm{B}\end{array}$ & $\begin{array}{l}140 \mathrm{NaCl} \\
\mathrm{mM}+10 \mathrm{mM} \mathrm{B}\end{array}$ \\
\hline NIAB- & $2.50 \mathrm{a} \pm$ & $1.80 \mathrm{~d} \pm$ & $1.55 \mathrm{~g} \pm 0.023$ & $1.40 \mathrm{j} \pm 0.005$ & $1.291 \pm 0.023$ & $1.20 \mathrm{~m} \pm 0.005$ & $1.14 \mathrm{no} \pm 0.011$ & $1.00 \mathrm{p} \pm 0.011$ & $0.80 \mathrm{~s} \pm 0.005$ \\
777 & 0.011 & $0.017(28 \%)$ & $(38 \%)$ & $(44 \%)$ & $(48.4 \%)$ & $(52 \%)$ & $(54.4 \%)$ & $(60 \%)$ & $(68 \%)$ \\
$\mathrm{IUB}-222$ & $2.52 \mathrm{a} \pm$ & $1.70 \mathrm{e} \pm 0.017$ & $1.40 \mathrm{j} \pm 0.023$ & $1.32 \mathrm{kl} \pm 0.003$ & $1.15 \mathrm{n} \pm 0.011$ & $1.00 \mathrm{p} \pm 0.017$ & $0.90 \mathrm{q} \pm 0.011$ & $0.74 \mathrm{t} \pm 0.017$ & $0.60 \mathrm{u} \pm 0.023$ \\
& 0.005 & $(32.53 \%)$ & $(44.44 \%)$ & $(47.61 \%)$ & $(54.36 \%)$ & $(60.31 \%)$ & $(64.28 \%)$ & $(70.63 \%)$ & $(76.19 \%)$ \\
$\mathrm{FH}-114$ & $2.53 \mathrm{~b} \pm$ & $2.07 \mathrm{c} \pm 0.028$ & $1.82 \mathrm{~d} \pm 0.005$ & $1.70 \mathrm{e} \pm 0.011$ & $1.55 \mathrm{~g} \pm 0.017$ & $1.47 \mathrm{hi} \pm 0.023$ & $1.35 \mathrm{k} \pm 0.005$ & $1.22 \mathrm{~m} \pm 0.011$ & $1.10 \mathrm{o} \pm 0.005$ \\
& 0.011 & $(18.18 \%)$ & $(28.05 \%)$ & $(32.80 \%)$ & $(38.73 \%)$ & $(41.89 \%)$ & $(46.64 \%)$ & $(51.77 \%)$ & $(56.52 \%)$ \\
$\mathrm{FH}-113$ & $2.45 \mathrm{a} \pm$ & $1.60 \mathrm{f} \pm 0.017$ & $1.33 \mathrm{kl} \pm 0.011$ & $1.20 \mathrm{~m} \pm 0.017$ & $1.00 \mathrm{p} \pm 0.023$ & $0.90 \mathrm{q} \pm 0.011$ & $0.80 \mathrm{~s} \pm 0.005$ & $0.60 \mathrm{u} \pm 0.017$ & $0.55 \mathrm{v} \pm 0.023$ \\
& 0.005 & $(34.69 \%)$ & $(45.71 \%)$ & $(51.08 \%)$ & $(59.18 \%)$ & $(63.26 \%)$ & $(67.34 \%)$ & $(75.51 \%)$ & $(77.55 \%)$ \\
$\mathrm{NIAB}-$ & $2.50 \mathrm{a} \pm$ & $1.45 \mathrm{i} \pm 0.017$ & $1.00 \mathrm{p} \pm 0.023$ & $0.85 \mathrm{r} \pm 0.011$ & $0.80 \mathrm{~s} \pm 0.017$ & $0.72 \mathrm{t} \pm 0.005$ & $0.55 \mathrm{v} \pm 0.011$ & $0.40 \mathrm{w} \pm 0.005$ & $0.20 \mathrm{x} \pm 0.005$ \\
846 & 0.005 & $(42 \%)$ & $(60 \%)$ & $(66 \%)$ & $(68 \%)$ & $(71.2 \%)$ & $(78 \%)$ & $(84 \%)$ & $(92 \%)$ \\
SGI-AA & $2.49 \mathrm{ab} \pm$ & $1.74 \mathrm{e} \pm 0.005$ & $1.50 \mathrm{~h} \pm 0.023$ & $1.33 \mathrm{k} \pm 0.005$ & $1.18 \mathrm{mn} \pm 0.017$ & $1.02 \mathrm{p} \pm 0.011$ & $0.90 \mathrm{q} \pm 0.011$ & $0.70 \mathrm{t} \pm 0.017$ & $0.63 \mathrm{u} \pm 0.005$ \\
& 0.017 & $(31.12 \%)$ & $(39.75 \%)$ & $(46.58 \%)$ & $(52 \%)$ & $(59.03 \%)$ & $(63.85 \%)$ & $(71.88 \%)$ & $(74.69 \%)$ \\
\hline
\end{tabular}

Table 2: Root dry weight of different cotton varieties $\left(\mathrm{g}_{\text {plant }}{ }^{-1}\right)$ at various salinity and boron levels

\begin{tabular}{|c|c|c|c|c|c|c|c|c|c|}
\hline Varieties & Control & $70 \mathrm{mM} \mathrm{NaCl}$ & $140 \mathrm{mM} \mathrm{NaCl}$ & $15 \mathrm{mM} \mathrm{B}$ & $10 \mathrm{mM} \mathrm{B}$ & $\begin{array}{l}70 \mathrm{~m} \mathrm{M} \\
\mathrm{NaCl}+5 \mathrm{~m} \mathrm{M} \mathrm{B}\end{array}$ & $\begin{array}{l}70 \mathrm{mM} \\
\mathrm{NaCl}+10 \mathrm{~m} \mathrm{M} \mathrm{B}\end{array}$ & $\begin{array}{l}140 \mathrm{mM} \\
\mathrm{NaCl}+5 \mathrm{~m} \mathrm{M} \mathrm{B}\end{array}$ & $\begin{array}{l}140 \mathrm{mM} \\
\mathrm{NaCl}+10 \mathrm{~m} \mathrm{M} \mathrm{B}\end{array}$ \\
\hline NIAB-777 & $\begin{array}{l}0.30 \mathrm{a} \pm \\
0.005\end{array}$ & $\begin{array}{l}0.21 \mathrm{c} \pm \\
0.005(30 \%)\end{array}$ & $\begin{array}{l}0.19 \text { cde } \pm \\
0.011(36.66 \%)\end{array}$ & $\begin{array}{l}0.17 \mathrm{efg} \pm \\
0.011(43.33 \%)\end{array}$ & $\begin{array}{l}0.156 \text { fgh } \pm \\
0.005(50 \%)\end{array}$ & $\begin{array}{l}0.14 \mathrm{hij} \pm \\
0.011(53.33 \%)\end{array}$ & $\begin{array}{l}0.13 \mathrm{ijk} \pm \\
0.011(56.66 \%)\end{array}$ & $\begin{array}{l}0.11 \mathrm{klm} \pm \\
0.005(63.33 \%)\end{array}$ & $\begin{array}{l}0.085 \mathrm{mno} \pm \\
0.006(73.33 \%)\end{array}$ \\
\hline IUB-222 & $\begin{array}{l}0.29 \mathrm{a} \pm \\
0.011\end{array}$ & $\begin{array}{l}0.2 \mathrm{~cd} \pm \\
0.011(31.03 \%)\end{array}$ & $\begin{array}{l}0.18 \mathrm{def} \pm \\
0.005(37.93 \%)\end{array}$ & $\begin{array}{l}0.15 \mathrm{ghi} \pm \\
0.005(48.27 \%)\end{array}$ & $\begin{array}{l}0.13 \mathrm{ijk} \pm \\
0.005(54.17 \%)\end{array}$ & $\begin{array}{l}0.12 \mathrm{jkl} \pm \\
0.005(58.62 \%)\end{array}$ & $\begin{array}{l}0.10 \operatorname{lmn} \pm \\
0.011(65.51 \%)\end{array}$ & $\begin{array}{l}0.10 \operatorname{lmn} \pm \\
0.005(55.55 \%)\end{array}$ & $\begin{array}{l}0.072 \mathrm{opq} \pm \\
0.005(75.17 \%)\end{array}$ \\
\hline FH-114 & $\begin{array}{l}0.30 \mathrm{a} \pm \\
0.005\end{array}$ & $\begin{array}{l}0.24 \mathrm{~b} \pm \\
0.005(20 \%)\end{array}$ & $\begin{array}{l}0.22 \mathrm{c} \pm \\
0.011(20 \%)\end{array}$ & $\begin{array}{l}0.20 \mathrm{~cd} \pm \\
0.005(33.33 \%)\end{array}$ & $\begin{array}{l}0.19 \mathrm{cde} \pm \\
0.011(36.66 \%)\end{array}$ & $\begin{array}{l}0.178 \text { def } \pm \\
0.011(40.66 \%)\end{array}$ & $\begin{array}{l}0.159 \text { fgh } \pm \\
0.005(47 \%)\end{array}$ & $\begin{array}{l}0.13 \mathrm{ijk} \pm \\
0.011(56.66 \%)\end{array}$ & $\begin{array}{l}0.11 \mathrm{klm} \pm \\
0.005(63.33 \%)\end{array}$ \\
\hline FH-113 & $\begin{array}{l}0.28 \mathrm{a} \pm \\
0.011\end{array}$ & $\begin{array}{l}0.19 \mathrm{cde} \pm \\
0.011(32.14 \%)\end{array}$ & $\begin{array}{l}0.17 \text { efg } \pm \\
0.005(39.28 \%)\end{array}$ & $\begin{array}{l}0.14 \mathrm{hij} \pm \\
0.017(50 \%)\end{array}$ & $\begin{array}{l}0.12 \mathrm{jkl} \pm \\
0.017(57.14 \%)\end{array}$ & $\begin{array}{l}0.11 \mathrm{klm} \pm \\
0.005(60.71 \%)\end{array}$ & $\begin{array}{l}0.09 \mathrm{mno} \pm \\
0.005(67.85 \%)\end{array}$ & $\begin{array}{l}0.081 \text { nop } \pm \\
0.005(71.07 \%)\end{array}$ & $\begin{array}{l}0.051 \mathrm{qrs} \pm \\
0.005(81.78 \%)\end{array}$ \\
\hline NIAB-846 & $\begin{array}{l}0.28 \mathrm{a} \pm \\
0.005\end{array}$ & $\begin{array}{l}0.16 \mathrm{fgh} \pm \\
0.005(42.85 \%)\end{array}$ & $\begin{array}{l}0.112 \mathrm{klm} \pm \\
0.005(60 \%)\end{array}$ & $\begin{array}{l}0.097 \mathrm{lmn} \pm \\
0.017(65.35 \%)\end{array}$ & $\begin{array}{l}0.08 \text { nop } \pm \\
0.005(71.4 \%)\end{array}$ & $\begin{array}{l}0.054 \mathrm{qrs} \pm \\
0.011(80.71 \%)\end{array}$ & $\begin{array}{l}0.05 \mathrm{qrs} \pm \\
0.005(82.14 \%)\end{array}$ & $\begin{array}{l}0.04 \mathrm{rs} \pm \\
0.011(85.11 \%)\end{array}$ & $\begin{array}{l}0.031 \mathrm{~s} \pm \\
0.005(88.92 \%)\end{array}$ \\
\hline SGI-AA & $\begin{array}{l}0.29 \mathrm{a} \pm \\
0.005\end{array}$ & $\begin{array}{l}0.2 \mathrm{~cd} \pm \\
0.005(31.03 \%)\end{array}$ & $\begin{array}{l}0.17 \mathrm{efg} \pm \\
0.011(41.37 \%)\end{array}$ & $\begin{array}{l}0.145 \mathrm{ghi} \pm \\
0.011(50 \%)\end{array}$ & $\begin{array}{l}0.14 \mathrm{hij} \pm \\
0.005(51.72 \%)\end{array}$ & $\begin{array}{l}0.12 \mathrm{jkl} \pm \\
0.011(58.63 \%)\end{array}$ & $\begin{array}{l}0.1 \operatorname{lmn} \pm \\
0.005(65.51 \%)\end{array}$ & $\begin{array}{l}0.09 \mathrm{mno} \pm \\
0.005(68.96 \%)\end{array}$ & $\begin{array}{l}0.06 \mathrm{pqr} \pm \\
0.005(79.31 \%)\end{array}$ \\
\hline
\end{tabular}

Each value is an average of 3 replications \pm SE. Means sharing the same letters are statistically non significant at $P \leq 0.05$. Values in parenthesis are percent decrease with respect to control.

chlorophyll contents decreased significantly (Table 3). Chlorophyll contents were more in case of FH-114 under all stress treatments. On the other hand, NIAB-846 showed minimum chlorophyll contents under both stresses. The percentage reduction in chlorophyll contents was 52 and $84 \%$ in FH-114 and NIAB-846 respectively, at $140 \mathrm{mM}$ $\mathrm{NaCl}+10 \mathrm{mM}$ B.

\section{Ionic Composition}

Salinity and boron stress significantly increased the shoot $\mathrm{Na}^{+}$and $\mathrm{B}$ concentration in all the varieties (Table 4 and 5). The individual as well as interactive effect of treatment and variety was found significant at $\mathrm{p} \leq 0.05$. The comparison of various treatments showed that shoot $\mathrm{Na}^{+}$concentration increased as the salinity level increased. The addition of B to saline conditions further enhanced this concentration in all the varieties. The concentration of $\mathrm{B}$ in all the varieties was more at higher B level as compared to lower B level. At the highest level of stress i.e. $140 \mathrm{mM} \mathrm{NaCl}+10 \mathrm{mM} \mathrm{B}$, FH-114 accumulated significantly lower $\mathrm{Na}^{+}\left(1.39 \mathrm{mmol} \mathrm{g}^{-1}\right.$ DW) and B (0.26 mmol g $\left.{ }^{-1} \mathrm{DW}\right)$ as compared to other varieties. On the other hand, maximum $\mathrm{Na}^{+}\left(1.94 \mathrm{mmol} \mathrm{g}^{-1}\right.$ DW) and B (0.49 mmol g $\left.{ }^{-1} \mathrm{DW}\right)$ concentrations were found in case of NIAB-846. The concentration of $\mathrm{K}^{+}$decreased significantly in response to both salinity and boron (Table $6)$. The individual effects of treatments, varieties and their interaction were found significant at $\mathrm{p} \leq 0.05$. In control treatment $\mathrm{K}^{+}$concentration was the maximum. Salinity and boron separately decreased $\mathrm{K}^{+}$concentration with more decrease in case of salinity alone. The highest reduction was noticed under the combined effect of highest salinity and boron levels. The comparison of varieties showed that at the highest stress level, significantly more $\mathrm{K}^{+}\left(0.45 \mathrm{mmol} \mathrm{g}^{-1}\right.$ DW) was accumulated by FH-114 and NIAB-846 accumulated significantly less $\mathrm{K}^{+}\left(0.2 \mathrm{mmol} \mathrm{g}^{-1} \mathrm{DW}\right)$ as compared to other varieties.

\section{Ranking of Varieties}

On the basis of growth and ionic parameters, quality points were assigned to the varieties on the basis of their performance under salinity and boron stress conditions. On the basis of these points the variety FH-114 attained (36) maximum points, followed by NIAB-777(30), IUB222(26), SG1-AA(20), FH-113(12) and NIAB-846(6) respectively. On the basis of these results, FH-114 was considered as a tolerant whereas NIAB-846 was considered as a sensitive to salinity and boron toxicity. 
Aftab et.al. / Int. J. Agirc. Biol., Vol. 17, No. 4, 2015

Table 3: Chlorophyll contents of different cotton varieties (SPAD value) at various salinity and boron levels

\begin{tabular}{|c|c|c|c|c|c|c|c|c|c|}
\hline Varieties & Control & $70 \mathrm{mM} \mathrm{NaCl}$ & $140 \mathrm{mM} \mathrm{NaCl}$ & $5 \mathrm{mM} \mathrm{B}$ & $10 \mathrm{mM} \mathrm{B}$ & $\begin{array}{l}70 \mathrm{mM} \\
\mathrm{NaCl}+5 \mathrm{mM} \mathrm{B}\end{array}$ & $\begin{array}{l}70 \mathrm{mM} \mathrm{NaCl}+1 \\
0 \mathrm{~m} \mathrm{M} \mathrm{B}\end{array}$ & $\begin{array}{l}140 \mathrm{mMNaCl}+5 \\
\mathrm{mM} \mathrm{B}\end{array}$ & $\begin{array}{l}140 \mathrm{mM} \mathrm{NaCl} \\
+10 \mathrm{~m} \mathrm{M} \mathrm{B}\end{array}$ \\
\hline NIAB-777 & $\begin{array}{l}40 \mathrm{abc} \pm \\
1.15\end{array}$ & $\begin{array}{l}35.4 \mathrm{de} \pm \\
1.039(11.5 \%)\end{array}$ & $\begin{array}{l}30 \mathrm{fg} \pm \\
0.577(25 \%)\end{array}$ & $\begin{array}{l}27.5 \mathrm{~h}-\mathrm{k} \pm \\
2.309(31.25 \%)\end{array}$ & $\begin{array}{l}24 \mathrm{k}-\mathrm{o} \pm \\
0.057(40 \%)\end{array}$ & $\begin{array}{l}22.1 \mathrm{n}-\mathrm{q} \pm \\
0.577(44.75 \%)\end{array}$ & $\begin{array}{l}19.3 \mathrm{rst} \pm 0.577 \\
(51.71 \%)\end{array}$ & $\begin{array}{l}17 \text { tuv } \pm \\
1.732(57.5 \%)\end{array}$ & $\begin{array}{l}14.5 \mathrm{vwx} \pm \\
0.288(63.7 \%)\end{array}$ \\
\hline IUB-222 & $\begin{array}{l}39.3 \mathrm{abc} \pm \\
1.301\end{array}$ & $\begin{array}{l}28.9 \mathrm{~g}-\mathrm{j} \pm \\
0.577(26.46 \%)\end{array}$ & $\begin{array}{l}25 \mathrm{j}-\mathrm{n} \pm \\
1.154(10.94 \%)\end{array}$ & $\begin{array}{l}22.8 \mathrm{~m}-\mathrm{q} \pm \\
1.154(41.98 \%)\end{array}$ & $\begin{array}{l}19.3 \text { rst } \pm \\
0.154(50.89 \%)\end{array}$ & $\begin{array}{l}\text { 17.4tuv } \pm \\
0.115(55.72 \%)\end{array}$ & $\begin{array}{l}14.6 \mathrm{vw} \pm 0.577 \\
(62.84 \%)\end{array}$ & $\begin{array}{l}13.6 w x y \pm \\
0.115(65.39 \%)\end{array}$ & $\begin{array}{l}11 \mathrm{yza} \pm 0.866 \\
(72.01 \%)\end{array}$ \\
\hline FH-114 & $\begin{array}{l}42 \mathrm{a} \pm \\
1.154\end{array}$ & $\begin{array}{l}39 \mathrm{abc} \pm \\
1.154(7.14 \%)\end{array}$ & $\begin{array}{l}37 \mathrm{cde} \pm \\
1.732(11.90 \%)\end{array}$ & $\begin{array}{l}35 \mathrm{ef} \pm \\
0.577(16.66 \%)\end{array}$ & $\begin{array}{l}32 \mathrm{fg} \pm \\
1.154(23.80 \%)\end{array}$ & $\begin{array}{l}29.5 \mathrm{gh} \pm \\
1.154(29.76 \%)\end{array}$ & $\begin{array}{l}26 \mathrm{i}-\mathrm{m} \pm 2.309 \\
(38.09 \%)\end{array}$ & $\begin{array}{l}21 \mathrm{o}-\mathrm{r} \pm \\
0.866(50 \%)\end{array}$ & $\begin{array}{l}20 \mathrm{q}-\mathrm{t} \pm 1.443 \\
(52.38 \%)\end{array}$ \\
\hline NIAB-846 & $\begin{array}{l}38.5 b c d \\
\pm 1.168\end{array}$ & $\begin{array}{l}20 \mathrm{q}-\mathrm{t} \pm \\
2.309(48.05 \%)\end{array}$ & $\begin{array}{l}18 \mathrm{stu} \pm \\
1.732(53.24 \%)\end{array}$ & $\begin{array}{l}14.4 \mathrm{vwx} \pm \\
1.154(62.59 \%)\end{array}$ & $\begin{array}{l}11.8 \mathrm{w}-\mathrm{z} \pm \\
1.115(69.35 \%)\end{array}$ & $\begin{array}{l}11.2 \mathrm{x}-\mathrm{a} \pm \\
1.115(70.90 \%)\end{array}$ & $\begin{array}{l}10 \mathrm{za} \pm 1.443 \\
(74 \%)\end{array}$ & $\begin{array}{l}8.20 \mathrm{ab} \pm \\
0.0577(78.70 \%)\end{array}$ & $\begin{array}{l}6 \mathrm{~b} \pm 0.577 \\
(84.41 \%)\end{array}$ \\
\hline SGI-AA & $\begin{array}{l}41.3 \mathrm{ab} \pm \\
2.482\end{array}$ & $\begin{array}{l}32 \mathrm{fg} \pm \\
1.154(22.51 \%)\end{array}$ & $\begin{array}{l}28 \mathrm{~h}-\mathrm{k} \pm \\
2.309(32.20 \%)\end{array}$ & $\begin{array}{l}26.2 \mathrm{i}-\mathrm{m} \pm \\
0.577(36.56 \%)\end{array}$ & $\begin{array}{l}22 \text { opqr } \pm \\
1.154(46.73 \%)\end{array}$ & $\begin{array}{l}19.3 \text { rst } \pm \\
0.981(53.26 \%)\end{array}$ & $\begin{array}{l}17 \text { tuv } \pm 0.288 \\
(58.83 \%)\end{array}$ & $\begin{array}{l}15 \text { uvw } \pm \\
1.732(63.68 \%)\end{array}$ & $\begin{array}{l}13 w-z \pm 0.577 \\
(68.52 \%)\end{array}$ \\
\hline
\end{tabular}

Each value is an average of 3 replications \pm SE. Means sharing the same letters are statistically non significant at $P \leq 0.05$. Values in parenthesis are percent decrease with respect to control.

Table 4: Shoot sodium concentration of different cotton varieties(mmol $\mathrm{g}^{-1} \mathrm{dry}$ weight) at various salinity and boron levels

\begin{tabular}{|c|c|c|c|c|c|c|c|c|c|}
\hline Varieties & Control & $\begin{array}{l}70 \mathrm{mM} \\
\mathrm{NaCl}\end{array}$ & $\begin{array}{l}140 \mathrm{mM} \\
\mathrm{NaCl}\end{array}$ & $5 \mathrm{mM} \mathrm{B}$ & $10 \mathrm{mM} \mathrm{B}$ & $\begin{array}{l}70 \mathrm{mM} \mathrm{NaCl}+5 \\
\mathrm{mM} \mathrm{B}\end{array}$ & $\begin{array}{l}70 \mathrm{mM} \mathrm{NaCl}+10 \\
\mathrm{mM} \mathrm{B}\end{array}$ & $\begin{array}{l}140 \mathrm{mM} \mathrm{NaCl}+ \\
5 \mathrm{mM} \mathrm{B}\end{array}$ & $\begin{array}{l}140 \mathrm{mM} \mathrm{NaCl} \\
+10 \mathrm{mM} \mathrm{B} \\
\end{array}$ \\
\hline NIAB-777 & $\begin{array}{l}0.14 \mathrm{c} \pm \\
0.011\end{array}$ & $\begin{array}{l}1 \mathrm{t} \pm \\
0.005\end{array}$ & $\begin{array}{l}1.26 \mathrm{op} \pm \\
0.014\end{array}$ & $\begin{array}{l}0.23 \mathrm{za} \pm \\
0.011\end{array}$ & $\begin{array}{l}0.27 \mathrm{yz} \pm \\
0.006\end{array}$ & $\begin{array}{l}1.22 \mathrm{pqr} \pm \\
0.028\end{array}$ & $\begin{array}{l}1.24 \mathrm{opq} \pm \\
0.023\end{array}$ & $\begin{array}{l}1.41 \mathrm{ijk} \pm \\
0.011\end{array}$ & $\begin{array}{l}1.47 \mathrm{fgh} \pm \\
0.017\end{array}$ \\
\hline FH-114 & $\begin{array}{c}0.12 \mathrm{c} \pm \\
0.017\end{array}$ & $\begin{array}{l}0.9 \mathrm{u} \pm \\
0.011\end{array}$ & $\begin{array}{l}1.2 \mathrm{qr} \pm \\
0.005\end{array}$ & $\begin{array}{l}0.2 \mathrm{ab} \pm \\
0.017\end{array}$ & $\begin{array}{l}0.24 \mathrm{za} \pm \\
0.011\end{array}$ & $\begin{array}{l}1.1 \mathrm{~s} \pm \\
0.017\end{array}$ & $\begin{array}{l}1.18 \mathrm{r} \pm \\
0.034\end{array}$ & $\begin{array}{l}1.32 \mathrm{mn} \pm \\
0.005\end{array}$ & $\begin{array}{l}1.39 \mathrm{kl} \pm \\
0.028\end{array}$ \\
\hline FH-113 & $\begin{array}{c}0.13 c \pm \\
0.006\end{array}$ & $\begin{array}{l}1.22 \mathrm{pqr} \pm \\
0.017\end{array}$ & $\begin{array}{l}1.47 \mathrm{fgh} \pm \\
0.040\end{array}$ & $\begin{array}{l}0.28 x y z \pm \\
0.005\end{array}$ & $\begin{array}{l}0.37 \mathrm{w} \pm \\
0.011\end{array}$ & $\begin{array}{l}1.39 \mathrm{kl} \pm \\
0.034\end{array}$ & $\begin{array}{l}1.45 \mathrm{~g}-\mathrm{j} \pm \\
0.005\end{array}$ & $\begin{array}{l}1.55 \mathrm{de} \pm \\
0.017\end{array}$ & $\begin{array}{l}1.71 b \pm \\
0.023\end{array}$ \\
\hline NIAB-846 & $\begin{array}{l}0.15 b c \\
\pm 0.023\end{array}$ & $\begin{array}{l}1.351 \mathrm{~m} \pm \\
0.011\end{array}$ & $\begin{array}{l}1.6 \mathrm{~cd} \pm \\
0.005\end{array}$ & $\begin{array}{l}0.33 w x \pm \\
0.011\end{array}$ & $\begin{array}{l}0.43 v \pm \\
0.023\end{array}$ & $\begin{array}{l}1.52 \mathrm{ef} \pm \\
0.011\end{array}$ & $1.6 \mathrm{~cd} \pm 0.011$ & $\begin{array}{l}1.71 b \pm \\
0.020\end{array}$ & $\begin{array}{l}1.94 \mathrm{a} \pm \\
0.023\end{array}$ \\
\hline SGI-AA & $\begin{array}{l}0.12 \mathrm{c} \pm \\
0.011\end{array}$ & $\begin{array}{l}1.2 \mathrm{qr} \pm \\
0.028\end{array}$ & $\begin{array}{l}1.4 \mathrm{jkl} \pm \\
0.017\end{array}$ & $\begin{array}{l}0.26 \mathrm{yz} \pm \\
0.011\end{array}$ & $\begin{array}{l}0.33 w x \pm \\
0.017\end{array}$ & $\begin{array}{l}1.31 \mathrm{mn} \pm \\
0.005\end{array}$ & $\begin{array}{l}1.46 \mathrm{ghi} \pm \\
0.011\end{array}$ & $\begin{array}{l}1.5 \mathrm{efg} \pm \\
0.028\end{array}$ & $\begin{array}{l}1.62 \mathrm{c} \pm \\
0.005\end{array}$ \\
\hline
\end{tabular}

Each value is an average of $3 \pm$ SE replications. Means sharing the same letters are statistically non significant at $P \leq 0.05$.

Table 5: Shoot boron concentration of different cotton varieties ( $\mathrm{mmol} \mathrm{g}^{-1}$ dry weight) at various salinity and boron levels

\begin{tabular}{|c|c|c|c|c|c|c|c|c|c|}
\hline Varieties & control & $70 \mathrm{mMNacl}$ & $140 \mathrm{mMNacl}$ & $5 \mathrm{mM} \mathrm{B}$ & $10 \mathrm{mM} B$ & $\begin{array}{l}70 \mathrm{~m} \mathrm{M} \\
\mathrm{NaCl}+5 \mathrm{mM} \mathrm{B}\end{array}$ & $\begin{array}{l}70 \mathrm{~m} \mathrm{M}+10 \mathrm{~m} \\
\mathrm{M} \mathrm{B}\end{array}$ & $\begin{array}{l}140 \mathrm{mM} \mathrm{NaCl}+5 \\
\mathrm{mM} \mathrm{B}\end{array}$ & $\begin{array}{l}140 \mathrm{mM} \mathrm{NaCl}+10 \\
\mathrm{mM} \mathrm{B}\end{array}$ \\
\hline \multirow[t]{2}{*}{ NIAB-777 } & $0.015 \mathrm{z} \pm$ & $0.029 x y z \pm$ & $0.058 \mathrm{u}-\mathrm{y} \pm$ & 0.166 rst \pm & 0.19 pqr \pm & $0.2 \mathrm{o}-\mathrm{r} \pm$ & $0.23 \mathrm{no} \pm$ & $0.271 \mathrm{~m} \pm$ & $0.3 \mathrm{jkl} \pm$ \\
\hline & 0.001 & 0.001 & 0.002 & 0.002 & 0.005 & 0.017 & 0.005 & 0.023 & 0.011 \\
\hline IUB-222 & 0.001 & 0.001 & 0.005 & 0.001 & 0.028 & 0.023 & 0.020 & 0.005 & 0.023 \\
\hline \multirow[t]{2}{*}{ FH-114 } & $0.015 \mathrm{z} \pm$ & $0.021 \mathrm{yz} \pm$ & $0.042 \mathrm{v}-\mathrm{z} \pm$ & $0.135 \mathrm{t} \pm$ & $0.15 \mathrm{st} \pm$ & $0.18 \mathrm{qrs} \pm$ & $0.19 \mathrm{pqr} \pm$ & $0.22 \mathrm{op} \pm$ & $0.26 \mathrm{mn} \pm$ \\
\hline & 0.0005 & 0.001 & 0.001 & 0.002 & 0.017 & 0.005 & 0.011 & 0.028 & 0.017 \\
\hline \multirow[t]{2}{*}{ FH-113 } & $0.016 \mathrm{z} \pm$ & $0.037 \mathrm{v}-\mathrm{z} \pm$ & $0.072 \mathrm{uv} \pm$ & $0.2050 \mathrm{pq} \pm$ & $0.3 \mathrm{jkl} \pm$ & $0.31 \mathrm{ijk} \pm$ & $0.35 \mathrm{fgh} \pm$ & $0.39 \mathrm{de} \pm$ & $0.44 b c \pm$ \\
\hline & 0.001 & 0.002 & 0.001 & 0.001 & 0.005 & 0.005 & 0.028 & 0.023 & 0.023 \\
\hline \multirow[t]{2}{*}{ NIAB-846 } & $0.015 \mathrm{z} \pm$ & $0.042 \mathrm{v}-\mathrm{z} \pm$ & $0.085 \mathrm{u} \pm$ & $0.236 \mathrm{mno} \pm$ & 0.34ghi \pm & 0.37 efg \pm & $0.42 \mathrm{~cd} \pm$ & $0.46 \mathrm{ab} \pm$ & $0.49 \mathrm{a} \pm$ \\
\hline & 0.0005 & 0.001 & 0.002 & 0.001 & 0.017 & 0.005 & 0.011 & 0.028 & 0.017 \\
\hline \multirow[t]{2}{*}{ SGI-AA } & $0.017 \mathrm{z} \pm$ & $0.035 \mathrm{v}-\mathrm{z} \pm$ & $0.068 \mathrm{uvw} \pm$ & $0.187 \mathrm{p}-\mathrm{s} \pm$ & $0.26 \mathrm{mn} \pm$ & $0.3 \mathrm{jkl} \pm$ & $0.31 \mathrm{ijk} \pm$ & 0.37 efg \pm & $0.38 \mathrm{ef} \pm$ \\
\hline & 0.002 & 0.001 & 0.002 & 0.0005 & 0.011 & 0.017 & 0.005 & 0.023 & 0.011 \\
\hline
\end{tabular}

Each value is an average of 3 replications \pm SE. Means sharing the same letters are statistically non significant at $P \leq 0.05$.

\section{Discussion}

This study revealed relatively high salinity and boron tolerance potential of the six cotton varieties. However, there was a distinct physicochemical changes occured in these six cotton varieties during study there behaviour with respect to salinity and boron toxicity tolerance. Shoot and root dry weights of all six varieties declined with the increasing levels of salinity and boron. Under all $\mathrm{NaCl}$ and B levels, NIAB-846 was more affected however FH-114 was less affected compared to rest of the varieties. The B stress alone was more detrimental than $\mathrm{NaCl}$ stress alone. Reduction in plant growth under salinity (Abbas et al., 2013) and combined stress of salinity and B (Ahmed, 2012) has previously been reported as well. Reduction in the growth of plants due to salinity is mainly attributed to three principle factors i.e osmotic effects, ion toxicity and deficiency of necessary nutrients (Munns and Tester, 2008). These factors are operative at both cellular and whole plant level and affect all the metabolic activities of crop plants. At 
Salinity and Boron Interaction in Cotton / Int. J. Agric. Biol., Vol. 17, No. 4, 2015

Table 6: Shoot potassium concentration of different cotton varieties ( $\mathrm{mmol} \mathrm{g}^{-1}$ dry weight) at various salinity and boron levels

\begin{tabular}{|c|c|c|c|c|c|c|c|c|c|}
\hline Varieties & Control & $70 \mathrm{mM} \mathrm{Nacl}$ & $140 \mathrm{mM} \mathrm{Nacl}$ & $5 \mathrm{mM} \mathrm{B}$ & $10 \mathrm{mM} \mathrm{B}$ & $70 \mathrm{~m} \mathrm{M}+5 \mathrm{~m} \mathrm{M} \mathrm{B}$ & $\begin{array}{l}70 \mathrm{mM} \mathrm{NaCl}+10 \\
\mathrm{mM} \mathrm{B}\end{array}$ & $\begin{array}{l}140 \mathrm{~m} \mathrm{M}+5 \\
\mathrm{~m} \mathrm{M} \mathrm{B}\end{array}$ & $\begin{array}{l}140 \mathrm{mM} \\
\mathrm{NaCl}+10 \mathrm{mM} \mathrm{B}\end{array}$ \\
\hline \multirow[t]{2}{*}{ NIAB-777 } & $1.54 \mathrm{a} \pm$ & $1 \mathrm{de} \pm$ & $0.74 \mathrm{ij} \pm$ & $1.1 \mathrm{c} \pm$ & $0.78 \mathrm{i} \pm$ & $0.55 \mathrm{mno} \pm$ & $0.50-r \pm$ & $0.45 \mathrm{qrs} \pm$ & $0.37 \mathrm{uvw} \pm$ \\
\hline & 0.011 & 0.057 & 0.017 & 0.057 & 0.028 & 0.005 & 0.011 & 0.005 & 0.020 \\
\hline IUB-222 & 0.005 & 0.017 & 0.011 & 0.005 & 0.023 & $0.005^{1}$ & 0.011 & 0.028 & 0.005 \\
\hline \multirow[t]{2}{*}{ FH-114 } & $1.57 \mathrm{a} \pm$ & $1.1 \mathrm{c} \pm$ & $0.8 \mathrm{hi} \pm$ & $1.2 \mathrm{~b} \pm$ & $0.85 \mathrm{gh} \pm$ & $0.65 \mathrm{kl} \pm$ & $0.57 \mathrm{mn} \pm$ & $0.52 \mathrm{nop} \pm$ & $0.45 q r s \pm$ \\
\hline & 0.023 & 0.057 & 0.046 & 0.017 & 0.023 & 0.011 & 0.005 & 0.017 & 0.023 \\
\hline \multirow[t]{2}{*}{ FH-113 } & $1.53 \mathrm{a} \pm$ & $0.9 \mathrm{fg} \pm$ & $0.64 \mathrm{kl} \pm$ & $1 \mathrm{de} \pm$ & $0.67 \mathrm{k} \pm$ & $0.47 \mathrm{pqr} \pm$ & 0.4 stu \pm & $0.34 \mathrm{u}-\mathrm{x} \pm$ & $0.27 \mathrm{y} \pm$ \\
\hline & 0.005 & 0.017 & 0.023 & 0.034 & 0.005 & 0.011 & 0.023 & 0.005 & 0.028 \\
\hline \multirow[t]{2}{*}{ NIAB-846 } & $1.54 \mathrm{a} \pm$ & $0.75 \mathrm{ij} \pm$ & $0.55 \mathrm{mno} \pm$ & $0.85 \mathrm{gh} \pm$ & $0.61 \mathrm{~m} \pm$ & 0.4 stu \pm & $0.33 \mathrm{v}-\mathrm{y} \pm$ & $0.3 x y \pm$ & $0.2 \mathrm{z} \pm$ \\
\hline & 0.006 & 0.028 & 0.011 & 0.017 & 0.005 & 0.011 & 0.005 & 0.028 & 0.023 \\
\hline \multirow[t]{2}{*}{ SGI-AA } & $1.55 \mathrm{a} \pm$ & $0.9 \mathrm{fg} \pm$ & $0.66 \mathrm{kl} \pm$ & $1.05 \mathrm{~cd} \pm$ & $0.7 \mathrm{jk} \pm$ & $0.50-\mathrm{r} \pm$ & $0.44 \mathrm{rst} \pm$ & 0.38 tuv \pm & $0.3 x y \pm$ \\
\hline & 0.017 & 0.017 & 0.028 & 0.011 & 0.023 & 0.005 & 0.011 & 0.017 & 0.023 \\
\hline
\end{tabular}

Each value is an average of 3 replications \pm SE. Means sharing the same letters are statistically non significant at $P \leq 0.05$.

higher levels of stress both root formation (Kramer, 1983) and their elongation (Garg and Gupta, 1997) is reduced to a greater extent. The physical growth parameters are usually related to salinity tolerance potential of all crops at their early stages of growth so they are the tools of selection criteria for salinity tolerance (Abbas et al., 2013). Decline in biomass yield can be attributed to decrease in assimilates under limited water and nutrient supply to the photosynthetic organs in the presence of excessive trace elements. The negative impacts of high B on plant growth have also been reported by (Alpaslan and Gunes, 2001) and (Sotiropoulos et al., 2002). Excessive B negatively affects a number of processes in vascular plants such as chlorophyll contents of leaf, lignin and suberin level and root cell division (Reid, 2007). As a result, reduced shoots and roots growth is typical chracterstics of plants exposed to high boron levels (Nable et al., 1990).

Chlorophyll contents were reduced due to both salinity and $\mathrm{B}$ toxicity with the later one having more injurious effects than the former one. The comparison of varieties indicated that FH-114 showed higher chlorophyll contents and NIAB-846 showed lower chlorophyll contents as compared to the rest of the varieties. The decrease in chlorophyll contents may be due to aminolevulinic acid (ALA), a precusor of chlorophyll which is seriously impaired under salinity and boron toxicity conditions (Tewari and Tripathy, 1998). However, other studies have indicated that excessive $\mathrm{B}$ can disrupt membranous structures including grana by inducing lipid peroxidation (Luna et al., 1994).

The ionic composition revealed that shoot $\mathrm{Na}^{+}$and $\mathrm{B}$ concentration of all the varieties increased significantly in response to increasing salinity levels with more accumulation in NIAB-846 than other varieties. This high accumulation of $\mathrm{Na}^{+}$and $\mathrm{B}$ in the tissues corresponds to the lower growth and dry weights of NIAB-846. These results are also supported by the findings of (Ahmed, 2012; Abbas et al., 2013). The buildup of poisonous ions in plant tissues is thought to be the major factor of decline in growth under salinity stress (Muscolo et al., 2003). Moreover, $\mathrm{K}^{+}$has a key role in salt tolerance where uptake of $\mathrm{K}^{+}$is decreased by $\mathrm{Na}^{+}$(Fox and Guerinot, 1998). In this experiment, the reduction of $\mathrm{K}^{+}$concentration was found in shoot which indicated that $\mathrm{Na}^{+}$decreased the uptake of $\mathrm{K}^{+}$. Decreased $\mathrm{K}^{+}$ uptake in response to salinity was also observed by (Ahmed, 2012; Abbas et al., 2013). The plants that accumulate higher $\mathrm{K}^{+}$and lower $\mathrm{Na}^{+}$uptake potential are considered better tolerant to salinity stress (Saqib et al., 2005). Same was the case in our experiment where $\mathrm{FH}-114$ owing to higher $\mathrm{K}^{+}$ and lower $\mathrm{Na}^{+}$uptake showed better salt tolerance than NIAB-846. The presence of $\mathrm{B}$ in the growing medium further increased the $\mathrm{Na}^{+}$concentration in the shoot of all the varieites and made the conditions more adverse for plants survival. On the basis of ranking, these varieties could be arranged based on their performance in growth and ionic parameters the variety $\mathrm{FH}-114$ performed best in all studied parameters and attained (36), NIAB-777 (30), IUB222, (26), FH-113 (12) and NIAB-846 (6) points. On the basis of these points FH-114 was selected as a tolerent variety while NIAB-846 was consider as a sensetive to salinity and boron toxicity.

\section{Conclusion}

The salinity and B has deterimental affect on the growth of cotton but the variety $\mathrm{FH}-114$ has potential to generate good growth in combined stress environment. There was evident that the dry shoot weight of variety FH-114 was maximum (1.10 $\left.\mathrm{g} \mathrm{plant}^{-1}\right)$ when compared with other varieties. The lowest shoot dry weight $\left(0.20 \mathrm{~g} \mathrm{plant}^{-1}\right)$ was observed in NIAB-846 at high level of combined stress $(140 \mathrm{mM}+10$ $\mathrm{mM} \mathrm{B})$. This showed that percent reduction in growth of NIAB-846 was $(92 \%)$ while in case of FH-114 the growth reduction was $(57 \%)$, while the other varieties growth reduction trend was NIAB-777(68\%) > IUB-222(76\%) > SG1-AA $(75 \%)>$ FH-113(78\%) at higher level of combined stress Similar treand was observed in case of ionic paremeters FH-114 accmulate less $\mathrm{Na}^{+}$and $\mathrm{B}$ while acquired more $\mathrm{K}^{+}$than other varieties while inverse trend was observed in case of NIAB-846. It was concluded that 
FH-114 was tolerant to salinity and boron stress as compare to other varieties, whereas NIAB-846 found very sensitive to salinity and boron stress. All other varieties showed response in between the response of these two varieties. From these results it can also be concluded that screening of plant varieties against combined stress of salinity and B can be done under controlled conditions. The variety FH-114 can be used under salinity and boron stress coditions for further studies.

\section{Acknowledgement}

The authors highly acknowledge the Higher Education Commission of Pakistan for the financial support to carry out this research.

\section{References}

Abbas, G., M. Saqib, J. Akhtar and S.M.A. Basra, 2013. Salinity tolerance potential of two acacia species at early seedling stage. Pak. J. Agric. Sci., 50: 683-688

Ahmad,N., M. Ibrahim, A. Niaz and M.Y. Nadeem, 2005. Boron concentration in canal and tube well water used for irrigation in some villages of Faisalabad district. J. Agric. Res., 43: 33-38

Ahmed, K., 2012. Salinity and boron interaction in maize, Ph. D. Thesis, University of Agriculture Faisalabad, Pakistan

Ahmad, R.T., T.A. Malik, I.A. Khan and M.J.A. Jaskani, 2009. Genetic analysis of some morpho-physiological traits related to drought stress in cotton (Gossypium hirsutum). Int. J. Agric. Biol., 11: 235-240

Alpaslan, M. and A. Gunes, 2001. Interactive effects of boron and salinity stress on the growth, membrane permeability and mineral composition of tomato and cucumber plants. Plant Soil, 236: 123-128

Anonymous, 2007. Economic Survey of Pakistan. Ministry of Food, Agriculture and Livestock, Economic Wing, Islamabad, Pakistan

Ashraf, M., 2002. Salt tolerance of cotton: some new advances. Crit. Rev. Plant Sci., 21: 1-30

Ashraf, M. and M.R. Foolad, 2007. Improving plant abiotic-stress resistance by exogenous application of osmoprotectants glycine betaine and proline. J. Environ. Exp. Bot., 59: 206-216

Ashraf, M. and S. Ahmad, 2000. Influence of sodium chloride on ion accumulation, yield components, and fiber characteristics in salttolerant and salt-sensitive lines of cotton (Gossypium hirsutum L.). Field Crops Res., 66: 115-127

FAO, 2008. FAO Land and Plant Nutrient Management Service. http://www. FAO. org/ag/agl/spush

Fox, T.C. and M.L. Guerinot, 1998. Molecular biology of cation transport in plants. Ann. Rev. Plant Physiol. Plant Mol. Biol., 49: 669-696

Garg, B.K. and I.C. Gupta, 1997. Saline Wastelands Environment and Plant Growth, pp: 287. Jodhpur, India, Scientific Publishers

Grieve, C.M., J.A. Poss, S.R. Grattan, D.L. Suarez and T.E. Smith, 2010. The combined effects of salinity and excess boron on mineral ion relations in broccoli. J. Sci. Hortic., 125: 179-187

Hasnain, A., S. Mahmood, S. Akhtar, S.A. Malik and N. Bashir, 2011. Tolerance and toxicity levels of boron in mung bean (Vigna radiata L.) Walczek cultivars at early growth stages. Pak. J. Bot., 43: $1119-1125$
Hoagland, D.R. and D.I. Arnon, 1950. The water culture method for growing plant without soil. California Agric. Exp. Station Circular, 347: $1-32$

Kramer, D., 1983. The possible role of transfer cells in the adaptation of plants to salinity. Physiol. Plant., 58: 549-555

Loomis, W.D. and R.W. Durst, 1992. Chem. Biol. boron. Biol. Factors, 3: 229-239

Luna, C.M., C.A. Gonzalez and V.S. Trippi, 1994. Oxidative damage caused by an excess of copper in oat leaves. Plant Cell Physiol., 35 $11-15$

Malekani, K. and M.S. Cresser, 1998. Comparison of three methods for determining boron in soil, plant and water samples. Commun. Soil Sci. Plant Anal., 29: 285-304

Maas, E.V. and G.J. Hoffmann, 1977. Crop salt tolerant current assessment. J. Irrig. Drainage Div, ASCE., 103: 115-134

Mills, H.A. and J.B. Jones, 1996. Plant Analysis Hand Book 11. Micro Macro publishing, Athens, G.A., USA

Miwa, K. and T. Fujiwara, 2010. Boron transport in plants: Co-ordinated regulation of transporters. Ann. Bot., 105: 1103-1108

Munns, R. and M. Tester, 2008. Mechanisms of salinity tolerance. Ann. Rev. Plant Biol., 59: 651-681

Muscolo, A., M. Sidari and M.R. Panuccio, 2003. Tolerance of kikuyu grass to long term salt stress is associated with induction of antioxidant defenses. Plant Growth Regul., 41: 57-62

Nable, R.O., B. Cartwright, R.C. Lance, 1990. Genotypic differences in boron accumulation in barley: relative susceptibilities to boron deficiency and toxicity. In: Genetic Aspects of Plant Mineral Nutrition, pp: 243-251. N. El Bassam, M. Dambroth, B. Loughman (eds.). Kluwer Academic Publishers, Dordrecht, The Netherlands

Reid, R, 2007. Identification of boron transporter genes likely to be responsible for tolerance to boron toxicity in wheat and barley. Plant Cell Physiol., 48: 1673-1678

Reid, R.J., J.E. Hayes, A. Post, J.C.R. Stangoulis and R.D. Graham, 2004. A critical analysis of the causes of boron toxicity in plants. Plant Cell Environ., 25: 1405-1414

Reuter, D.J. and J.B. Robinson, 1986. Plant Analysis and Interpretation Manual. Inkata press, Melbourne, Australia

Saleem, M.Y., M. Khanif, F. Ishak, A.W. Samsuri and B. Hafeez, 2011. Importance of boron for agriculture productivity: A review. Int. Res. J. Agric. Sci. Soil Sci., 1: 293-300

Saqib, M., C. Zorb, Z. Rengel and S. Schubert, 2005. $\mathrm{Na}^{+}$exclusion and salt resistance of wheat (Triticum aestivum L.) are improved by the expression of endogenous vacuolar $\mathrm{Na}^{+} / \mathrm{H}^{+}$antiporters in roots and shoots. Plant Sci., 169: 959-965

Sestren, J.A. and R. Kroplin, 2009. Sintomas de toxicidade de boro no algodoeiro. In: VII Con-gresso Brasileiro De Algodão. ABRAPA/IAPAR, Foz do Iguaçu

Steel, R.G.D., J.H. Torrie and D.A. Dickey, 1997. Principles and Procedures of Statistics: A Biometrical Approach, 3rd edition McGraw Hill Co., New York, USA

Sotiropoulos, T.E., N.I. Therios, N.K. Dimassi, A. Bosbalidis and G. Kofilids, 2002. Nutritional status, growth, $\mathrm{CO}_{2}$ assimilation, and leaf anatomical responses in two kiwi fruit species under boron toxicity. J. Plant Nutr., 5: 1244-1261

Tewari, A.K. and B.C. Tripathy, 1998. Temperature-stress induced impairment of chlorophyll biosynthesis reactions in cucumber and wheat. Plant Physiol., 117: 851-858

(Received 29 September 2014; Accepted 20 December 2014) 